\title{
Dispersion-driven ferromagnetism in a flat-band Hubbard system
}

\author{
Oleg Derzhko ${ }^{1,2,3,4}$ and Johannes Richter ${ }^{1,5}$ \\ ${ }^{1}$ Max-Planck-Institut für Physik komplexer Systeme, Nöthnitzer Straße 38, 01187 Dresden, Germany \\ ${ }^{2}$ Institute for Condensed Matter Physics, National Academy of \\ Sciences of Ukraine, 1 Svientsitskii Street, L'viv-11, 79011, Ukraine \\ ${ }^{3}$ Department for Theoretical Physics, Ivan Franko National University of L'viv, 12 Drahomanov Street, L'viv-5, 79005, Ukraine \\ ${ }^{4}$ Abdus Salam International Centre for Theoretical Physics, Strada Costiera 11, I-34151 Trieste, Italy \\ ${ }^{5}$ Institut für theoretische Physik, Otto-von-Guericke-Universität Magdeburg, P.O. Box 4120, D-39016 Magdeburg, Germany
}

(Dated: October 2, 2018)

\begin{abstract}
We investigate a mechanism to establish ground-state ferromagnetism in flat-band Hubbard systems based on a kind of order-from-disorder effect driven by dispersion. As a paradigm we consider a frustrated diamond chain, where for ideal diamond geometry the lowest one-electron band is flat, but the ground state remains paramagnetic for arbitrary on-site repulsion $U$. We focus on half filling of the flat band. By using numerical and analytical arguments we present the ground-state phase diagram for a distorted diamond chain, i.e., the former flat band becomes dispersive. Driven by the interplay of dispersion and interaction the ground state is ferromagnetic if the interaction exceeds a critical value $U_{c}$.
\end{abstract}

PACS numbers: 71.10.-w, 75.10.Lp, 75.10.Jm

Keywords: Hubbard model, flat band, ferromagnetism

Electronic as well as localized-spin systems with dispersionless (i.e., flat) one-particle bands offer a unique playground in view of realizing unconventional lowtemperature phases, see, e.g., Refs. $1-6$ and references therein. An illustrious example is the fractional quantum Hall effect caused by interactions within the highly degenerate manifold of the dispersionless Landau levels ${ }^{7}$ Very recently, remarkably in flat-band systems hightemperature fractional quantum Hall states without a magnetic field were found, $\underline{\underline{1}}$ Relevant perturbations leading to dispersion of the one-particle band or interactions between the particles may yield highly non-trivial correlation effects. An intriguing example is the ferromagnetic instability in flat-band Hubbard models when the interaction prevails against the kinetic energy $\underline{\underline{8}} \underline{\underline{13}}$ This class of interacting quantum systems belongs to the notoriously rare examples where rigorous results for many-body ground states are available. After the seminal papers by Mielke and Tasaki ${ }^{-11}$ a continuous theoretical activity in this field can be recorded $\cong$ 2.12-34 Additional interest in this subject stems from the experimental side. Besides several promising materials realizing flat-band ferromagnetism (FM) $\stackrel{35-39}{\underline{3}}$ generated flat-band phases in optical lattices open a wide area for experimental research activities, see, e.g., Refs. 4042 .

A crucial point to understand the mechanism leading to flat-band FM is the existence of localized eigenstates, i.e., the particles can be localized within small trapping cells on a lattice see, e.g., Refs. 5, 6, , , 9, 29, 31. Thus many-electron ground states can be constructed by filling the traps obeying the Pauli principle, and a geometrical representation of the localized many-particle states is at hand. As a result relevant quantum degrees of freedom can be mapped on classical ones thus providing the powerful toolbox of classical statistical mechanics $29,31,43,44$ In addition, it is worth noticing that this mapping also opens a window to a new percolation problem, called Pauli-correlated percolation, in which the first-order nature of an equilibrium percolation transition can be established. $\stackrel{13,45}{ }$ These findings indicate that Hubbard flat-band systems are of broad conceptual relevance.

Based on the geometrical interpretation of the localized many-body states the saturated FM then corresponds to the full occupation of all trapping cells say with upelectrons $\frac{29}{2}$ Importantly, it is necessary to have overlapping traps (i.e., neighboring cells must share at least one site). Then even if all the cells are occupied with electrons having identical spin (symmetric spin state) they can avoid the Hubbard on-site repulsion $U>0$ and this fully polarized state remains within the ground-state manifold. However, in case that the trapping cells are isolated from each other there is no route to FM and the large set of paramagnetic states prevails against the ferromagnetic eigenstate $\underline{24,31,33,34}$

Introducing dispersion (violation of the flat-band geometry) typically modifies the balance of interaction and kinetic energy and tends to destabilize FM. However, it was demonstrated by several studies that the ferromagnetic ground state is robust if the flat band becomes (slightly) dispersive and the Hubbard repulsion $U$ is larger than a threshold $U_{c}>0$, where $U_{c}$ depends on the degree of the violation of the flat-band geometry $\underline{14}-16$

In the present paper we will demonstrate that unexpectedly for a certain class of flat-band systems having isolated trapping cells just the dispersion, i.e., the kinetic energy, will open the route to ferromagnetic ground states. This dispersion-driven FM resembles the celebrated order-from-disorder mechanism, $\stackrel{46.47}{ }$ i.e., due to distortions an ordered ground state is selected from the degenerate flat-band ground-state manifold where paramagnetic states dominate. 


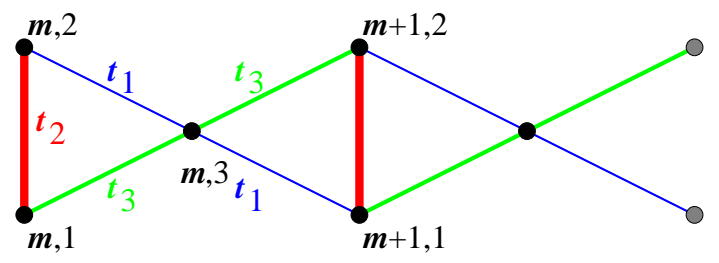

FIG. 1: (Color online) The frustrated diamond chain corresponding to the Hamiltonian (11). The trapping cells for localized electrons (vertical dimers) are indicated by bold red lines ( $t_{2}$ bonds). The lattice sites are labeled by a pair of indeces, where the first number enumerates the unit cells $(m=1, \ldots, \mathcal{N}, \mathcal{N}=N / 3$ is the number of unit cells) and the second one enumerates the position of the site within the unit cell.

We consider the standard repulsive Hubbard model

$$
H=\sum_{(i j), \sigma} t_{i j}\left(c_{i, \sigma}^{\dagger} c_{j, \sigma}+c_{j, \sigma}^{\dagger} c_{i, \sigma}\right)+U \sum_{i} n_{i, \uparrow} n_{i, \downarrow} .
$$

Here the sum in the hopping term runs over all nearest neighbors on the lattice, $\sigma=\uparrow, \downarrow, t_{i j}>0, c_{i, \sigma}^{\dagger}\left(c_{i, \sigma}\right)$ are the usual fermion creation (annihilation) operators, the sum in the interaction term runs over all $N$ lattice sites, and $n_{i, \sigma}=c_{i, \sigma}^{\dagger} c_{i, \sigma}$.

We focus on a generic example of the flat-band lattice with isolated trapping cells, namely the frustrated diamond-chain lattice, $\frac{31}{=}$ see Fig. 1 The flat-band case is realized for $t_{1}=t_{3}=t$. Then electrons can be localized within a characteristic trapping cell (vertical dimer) due to destructive quantum interference. The one-particle energy of the flat-band states is $\varepsilon_{1}=-t_{2}$, whereas the energies of the one-particle states from the two other dispersive bands are $\varepsilon_{2,3}(\kappa)=t_{2} / 2 \mp \sqrt{t_{2}^{2} / 4+4 t^{2}(1+\cos \kappa)}$. Here $\kappa=2 \pi m / \mathcal{N}, m \in \mathbb{Z},-\mathcal{N} / 2 \leq m<\mathcal{N} / 2$. The number of unit cells $\mathcal{N}=N / 3$ equals the number of trapping cells. The two dispersive bands have the same widths $w_{2,3}=\sqrt{t_{2}^{2} / 4+8 t^{2}}-t_{2} / 2 \approx 2\left(t_{3}+t_{1}\right)^{2} / t_{2}$ if $t_{2} / t \gg 1$. The flat-band states are the lowest-energy ones with a gap to excited states $\varepsilon_{2}(0)-\varepsilon_{1}>0$ if $t_{2}>2 t$.

Owing to the localized nature of the one-electron flatband states the many-electron states in the subspaces with $n=2, \ldots, \mathcal{N}$ electrons for $U>0$ can be constructed by filling the traps arbitrarily with up- or down-spin electrons leading to macroscopic ground state degeneracy of $2^{n} \mathcal{C}_{\mathcal{N}}^{n}$, where the paramagnetic states are predominant. Clearly all these states are linearly independent $\underline{\underline{48}}$ Moreover, these localized many-electron states have the lowest energy in their corresponding $n$-electron subspaces, if the flat band is the lowest, i.e., for $t_{2}>2 t$.

Now we focus on an electron filling corresponding to a half-filled flat band, i.e., $n=\mathcal{N}$. Then all traps are occupied with precisely one electron with arbitrary spin orientation, and the intermediate sites out of the trapping cells are empty. The ground states exhibit perfect charge order, but the averaged magnetic moment per site at $T=$
0 is zero for $N \rightarrow \infty \stackrel{31}{\underline{31}}$ Moreover, these charge-ordered ground states are protected by a charge gap.

A relevant violation of the ideal flat-band geometry is known from the fascinating frustrated magnetic compound azurite $\underline{\underline{49}}$ We adopt the azurite geometry and consider different values of $t_{1}$ and $t_{3}$ but fixing their average, i.e., $t_{1} \neq t_{3}, t_{1}+t_{3}=2 t$, see Fig. 1. Moreover, we assume $t_{2}>2 t$, see above. For $t_{1} \neq t_{3}$ the lowestenergy band acquires dispersion and its band width becomes $W_{1} \approx 2\left(t_{3}-t_{1}\right)^{2} / t_{2}$. The ratio between $W_{1}$ and the band width $w_{2,3}$ of the upper dispersive bands

$$
\frac{W_{1}}{w_{2,3}} \approx \Omega^{2}, \quad \Omega \equiv\left|\frac{t_{3}-t_{1}}{t_{3}+t_{1}}\right|
$$

gives an appropriate dimensionless parameter $\Omega^{2}$ that characterizes the acquired dispersion of the former flat band and which, in turn, can be used to measure the strength of deviation from the ideal flat-band geometry.

Our main result obtained by exact diagonalization (ED) and fourth-order perturbation theory (PT) is compactly illustrated by the phase diagram in Fig. 2, For not too large deviations from ideal geometry controlled by $\Omega^{2}<1$ and for sufficiently large $U / t_{2}>U_{c} / t_{2}$, the ground state of the Hubbard diamond chain with halffilled lowest band is ferromagnetic (the region denoted as "FM").

Let us now illustrate briefly our calculations leading to Fig. 2. The Lanczos ED to calculate the ground states was performed for chains of $N=12,18,24$ sites, i.e., of $\mathcal{N}=4,6,8$ unit cells, with periodic boundary conditions imposed. We set $t_{3}+t_{1}=2$ and consider various values of $t_{2}$. In Fig. 2 we present data for $t_{2}=3$ and 6 . The parameter characterizing the dispersion was varied from $t_{3}-t_{1}=0\left(\Omega^{2}=0\right)$ to $t_{3}-t_{1}=2\left(\Omega^{2}=1\right)$. For quite (but not arbitrarily) large deviations from ideal geometry $\Omega^{2}$ and sufficiently large $U>U_{c}$ there is a unique ferromagnetic ground state, i.e., the ground state is a ferromagnetic $\mathrm{SU}(2)$ multiplet which has the degeneracy $n+1=\mathcal{N}+1$. In the limit $U \rightarrow \infty$ there is a maximal dispersion $\Omega_{c}^{2}$ above which ground-state FM does not exist. As expected there is a finite-size dependence of $U_{c}\left(\Omega^{2}\right)$, but the general shape of the curve is the same for all $N$. Interestingly the region of FM increases with growing system size $N$. Thus, for $N=12,18,24$, and 30 sites with $t_{2}=3$ we found in the limit $U \rightarrow \infty$ the values $\Omega_{c}^{2} \approx 0.354,0.591,0.778$, and 0.884 , respectively. That gives clear evidence that the dispersion-driven FM exists for $N \rightarrow \infty$. The influence of the vertical hopping integral $t_{2}$ on the phase boundary $U_{c}\left(\Omega^{2}\right)$ is visible from the corresponding curves for $N=18$. For small band width $\Omega^{2}$ the region of FM slightly shrinks with increasing of $t_{2}$, whereas $U_{c}$ grows with increasing $t_{2}$ for $\Omega^{2} \gtrsim 0.25$.

Let us finally mention, that the dispersion does not change substantially the charge order present for the ideal flat-band geometry. For example, for $N=18, U=10$, $t_{2}=3, \Omega^{2}=0.25$ the occupation of the intermediate sites amounts less than $10 \%$ of the occupation of the sites of the trapping cells. 

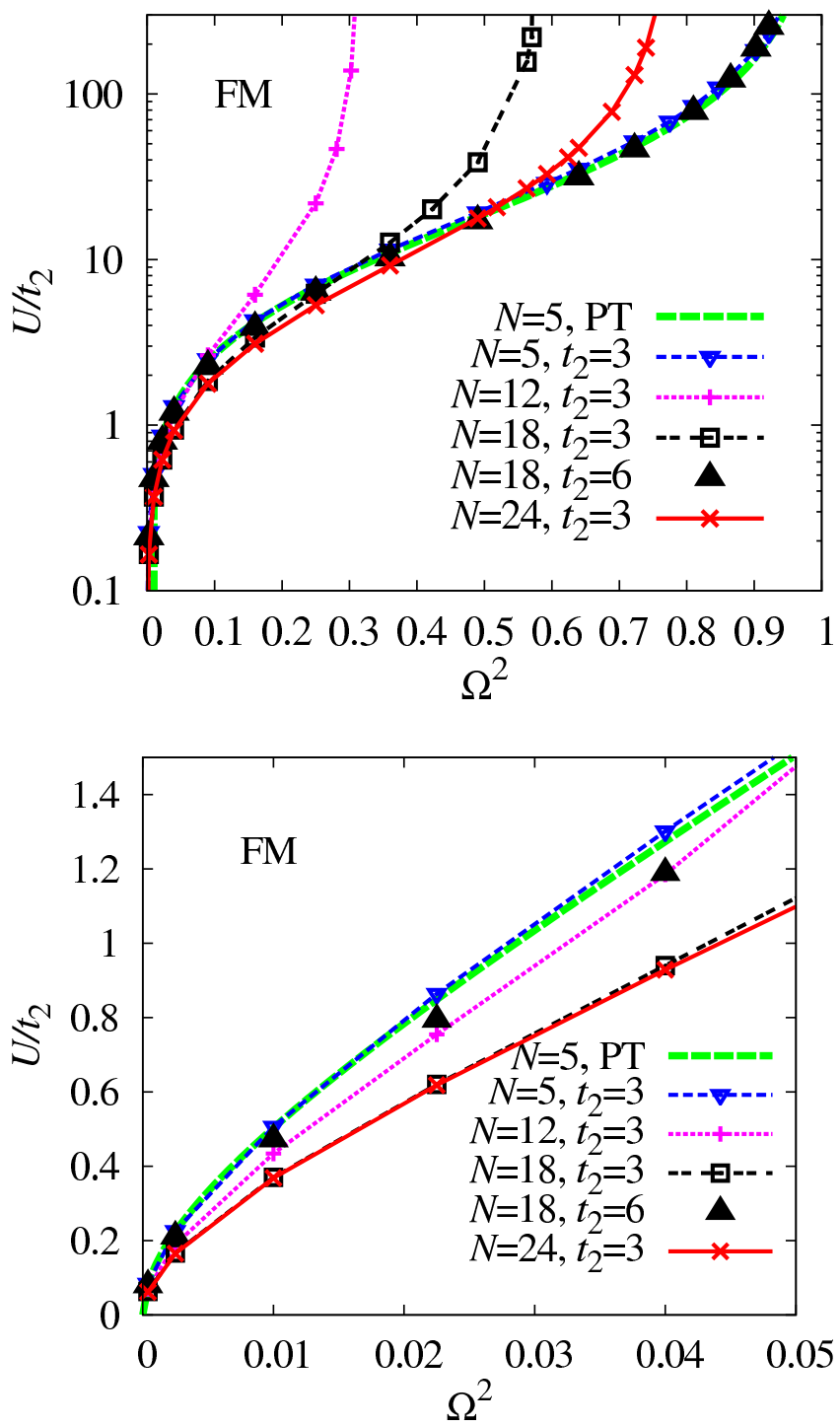

FIG. 2: (Color online) Phase diagram of the distorted Hubbard diamond chain with electron density $n / N=1 / 3$ (halffilled lowest band). Ferromagnetism (denoted by "FM") appears for on-site repulsions $U$ above a critical value $U_{c} . U_{c}$ is shown as a function of the dimensionless band-width parameter $\Omega^{2}$, see Eq. (2). The various critical lines $U_{c}\left(\Omega^{2}\right)$ are obtained by fourth-order perturbation theory (symbol "PT"), see Eq. (5), and by exact diagonalization for finite systems of $N=5$ (open boundary conditions) and $N=12,18,24$ (periodic boundary conditions) for hopping parameter sets with $t_{3}+t_{1}=2$ and $t_{2}=3,6$.

In the next step we complement our numerical data by fourth-order PT. We will illustrate only some main features of this approach. More details can be found in the Appendix. In fourth order it is sufficient to consider a 5 -site cluster that contains two trapping cells (the sites $m, 1, m, 2, m, 3, m+1,1$, and $m+1,2$ connected by six bonds in Fig. 10 in the subspace of $n=2$ electrons. We apply standard $\mathrm{PT} \underline{\underline{50}} \underline{-53}$ The unperturbed
Hamiltonian contains just the $t_{2^{-}}$and $U$-terms (recall that $t_{2}>2 t=t_{3}+t_{1}$ is the dominating hopping integral). The perturbation contains the $t_{3^{-}}$and $t_{1}$-terms. The unperturbed ground states in the two-electron subspace with accounting $\mathrm{SU}(2)$ symmetry have the form $|t, 1\rangle=$ $l_{m, \uparrow}^{\dagger} l_{m+1, \uparrow}^{\dagger}|0\rangle,|t, 0\rangle=(1 / \sqrt{2})\left(l_{m, \uparrow}^{\dagger} l_{m+1, \downarrow}^{\dagger}+l_{m, \downarrow}^{\dagger} l_{m+1, \uparrow}^{\dagger}\right)|0\rangle$, $|t,-1\rangle=l_{m, \downarrow}^{\dagger} l_{m+1, \downarrow}^{\dagger}|0\rangle$ (the components of the triplet state), and $|s\rangle=(1 / \sqrt{2})\left(l_{m, \uparrow}^{\dagger} l_{m+1, \downarrow}^{\dagger}-l_{m, \downarrow}^{\dagger} l_{m+1, \uparrow}^{\dagger}\right)|0\rangle$ (the singlet state). Here $l_{m, \sigma}^{\dagger}=\left(c_{m, 1, \sigma}^{\dagger}-c_{m, 2, \sigma}^{\dagger}\right) / \sqrt{2}$ and $|0\rangle$ denotes the vacuum state.

Calculating the energy up to the fourth order we find for the triplet states

$$
\begin{array}{r}
E_{t}=-2 t_{2}-\frac{\left(t_{3}-t_{1}\right)^{2}}{t_{2}} \\
-\frac{\left(t_{3}+t_{1}\right)^{2}\left(t_{3}-t_{1}\right)^{2}}{2 t_{2}^{3}}+\frac{\left(t_{3}-t_{1}\right)^{4}}{t_{2}^{3}}+\ldots,
\end{array}
$$

i.e., $E_{t}$ is independent of $U$. The energy of the singlet state $E_{s}$ depends on $U$. For the forth-order analytical expression for $E_{s}(U)$ see the Appendix. In the limit $U \rightarrow$ $\infty$ it becomes

$$
\begin{array}{r}
E_{s}(\infty)=-2 t_{2}-\frac{\left(t_{3}-t_{1}\right)^{2}}{t_{2}} \\
-\frac{\left(t_{3}+t_{1}\right)^{2}\left(t_{3}-t_{1}\right)^{2}}{4 t_{2}^{3}}+\frac{3\left(t_{3}-t_{1}\right)^{4}}{4 t_{2}^{3}}+\ldots
\end{array}
$$

and $E_{s}(\infty)>E_{t}$ due to the fourth-order term. Thus, the ground state in the limit $U \rightarrow \infty$ is ferromagnetic. In the small- $U$ limit, the dominating term in $E_{s}(U)$ is $-2\left(t_{3}-t_{1}\right)^{4} /\left(U t_{2}^{2}\right)$ which obviously leads to the opposite inequality $E_{s}(U)<E_{t}$, i.e., the ground state is nonmagnetic. To determine the critical value of $U_{c}$, above which the ground state is ferromagnetic we have to solve the equation $E_{t}=E_{s}\left(U_{c}\right)$ which gives

$$
\frac{U_{c}}{t_{2}}=\frac{\sqrt{16+65 \Omega^{2}}+9 \Omega}{1-\Omega^{2}} \Omega .
$$

Obviously, for small deviations from the flat-band case, i.e., $\Omega^{2} \ll 1$, we get $U_{c} / t_{2} \approx 4 \Omega$. The graphical representation of Eq. (5D) is shown by the thick long-dashed green line in Fig. 2. The effective Hamiltonian describing the low-energy degrees of freedom of the 5 -site two-electron Hubbard problem is the 2-site spin-1/2 Heisenberg model

$$
H_{\mathrm{eff}}=\mathrm{J}(U) \mathbf{T}_{m} \cdot \mathbf{T}_{m+1}+\mathrm{C}(U)
$$

where $\mathrm{J}(U)=E_{t}-E_{s}(U), \mathrm{C}(U)=\left[3 E_{t}+E_{s}(U)\right] / 4$. The pseudospin operators are given by $T_{m}^{+}=l_{m, \uparrow}^{\dagger} l_{m, \downarrow}$, $T_{m}^{-}=l_{m, \downarrow}^{\dagger} l_{m, \uparrow}$, and $T_{m}^{z}=\left(l_{m, \uparrow}^{\dagger} l_{m, \uparrow}-l_{m, \downarrow}^{\dagger} l_{m, \downarrow}\right) / 2$. The exchange constant $\mathrm{J}(U)$ in Eq. (6) is positive in the small$U$ limit, changes its sign at $U_{c}$ given by Eq. (5), and approaches $-t_{3} t_{1}\left(t_{3}-t_{1}\right)^{2} / t_{2}^{3}<0$ as $U \rightarrow \infty$. According to the PT the phase diagram is universal if we use $\Omega^{2}$ and $U / t_{2}$ for the axis, i.e., the data for $U_{c}$ for various $t_{1}$, 
$t_{2}, t_{3}$ should collapse to one universal curve (5). Another consequence of Eq. (5) is that $U_{c} \rightarrow \infty$ if $\Omega^{2} \rightarrow 1$, i.e., Eq. (5) yields $\Omega_{c}^{2}=1$. As a direct check of the fourthorder PT results for $E_{t}$ and $E_{s}(U)$ we have performed ED for the 5-site cluster, see the dashed blue line with empty down-triangles in Fig. 2 The agreement is excellent.

In summary, the PT confirms our ED results for the existence of FM. The quantitative agreement between $\mathrm{ED}$ and $\mathrm{PT}$ is the better the smaller $\Omega^{2}$ and the larger $t_{2}$. The appearance of ground-state FM for deviations from ideal geometry is a result of fourth-order processes. Higher-order PT naturally would enlarge the region of quantitative agreement with ED data.

For experimental research the existence of a charge gap that would protect the considered state against charge fluctuations is of relevance. As already mentioned above such a charge gap exists for the ideal geometry 31 To calculate the charge gap for the distorted geometry we consider the grand-canonical setup adding to the Hamiltonian (11) the term with a chemical potential, $-\mu \sum_{i}\left(n_{i, \uparrow}+n_{i, \downarrow}\right)$. In Fig. 3 we show the ground-state behavior of the averaged electron density (per cell) $\bar{n} / \mathcal{N}$ as a function of the chemical potential $\mu$. There is a wide plateau appearing for $U \gtrsim 1$ at $\bar{n} / \mathcal{N}=1$ that is almost independent of the distortion. For $U \lesssim 1$ the plateau is less pronounced and it disappears for $\widetilde{U}=0$. The width of the plateau $\Delta \mu$ corresponds to the size of the charge gap. Fig. 3 gives evidence that this charge gap is robust against the deviation from ideal flat-band geometry, and, thus, the protection works also in this case. Moreover, the finite-size dependence is small (see also Refs. 29.31).

To summarize, we have considered a dispersion-driven emergence of the ground-state FM in the Hubbard model on a frustrated diamond chain using ED and PT approaches. The ferromagnetic ground state is observed for half filling of the lowest one-particle band. It is protected by a charge gap against charge fluctuations and exhibits also charge ordering. The discussed scenario of dispersion-driven FM is not restricted to specific geometry of the frustrated diamond chain, rather it is quite general for lattices with isolated cells including two-dimensional ones. Indeed ED calculations confirm this expectations for the lattices considered in Ref. 31 .

Our focus on the diamond-chain geometry is motivated by the fact that this geometry is often found in natural compounds, see, e.g., Refs. 49,54,55. Its simplicity is advantageous in realizing this geometry, e.g., in optical lattices or quantum dot arrays. Since one may expect that it is hard to realize the perfect flat-band geometry in experiments the discussion of distortions is of crucial relevance.

There is an ongoing experimental search for flat-band ferromagnets. The possibility of flat-band FM in quantum dot arrays and in quantum atomic wires formed on solid surfaces was discussed in Ref. 35. Other examples for the flat-band ferromagnets come from polymers 37 where a search for purely organic ferromagnets is known as a challenging target. Another exper-

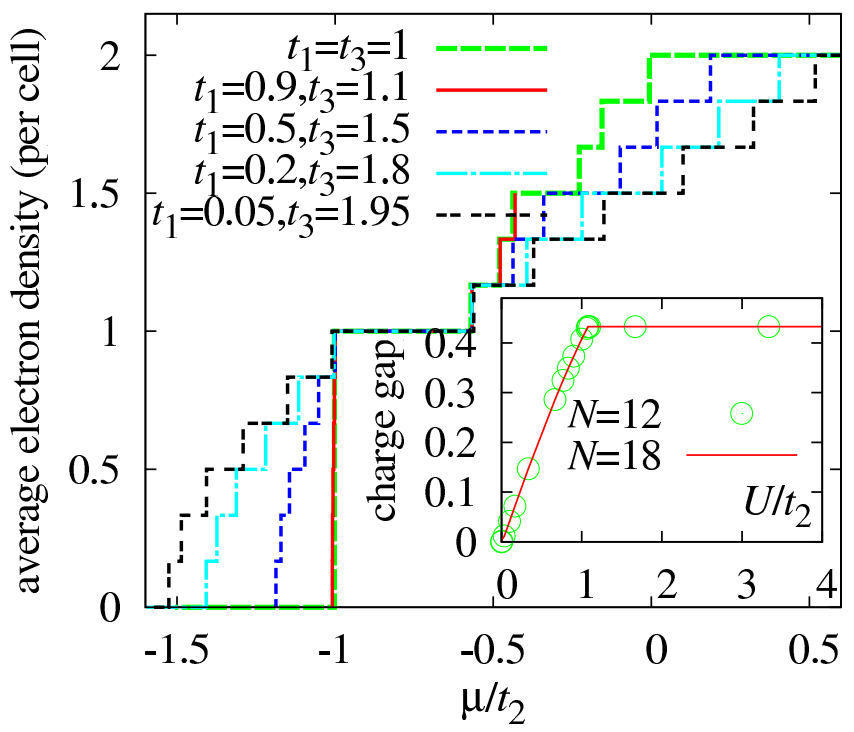

FIG. 3: (Color online) Averaged electron density (per cell) $\bar{n} / \mathcal{N}$ versus chemical potential $\mu / t_{2}$ for the frustrated diamond chain of $N=18$ sites with $t_{2}=3, t_{1}=t_{3}=1, t_{1}=0.9$, $t_{3}=1.1, t_{1}=0.5, t_{3}=1.5, t_{1}=0.2, t_{3}=1.8$, and $t_{1}=0.05$, $t_{3}=1.95$, and $U \rightarrow \infty$. Inset: Charge gap (plateau width) $\Delta \mu / t_{2}$ at $\bar{n} / \mathcal{N}=1$ versus $U / t_{2}$ for the distorted frustrated diamond chains of $N=12$ (circles) and $N=18$ (line) sites with $t_{2}=3, t_{1}=0.9, t_{3}=1.1$.

imental realization of the flat-band FM has been discussed recently in Ref. 39. Specific organic molecules [tetracyano- $p$-quinodimethane (TCNQ) molecules] deposited on graphene epitaxially grown on $\mathrm{Ru}(0001)$ acquire charge from the substrate and develop a magnetic moment which survives when the molecules form a monolayer. The authors of Ref. 39 claim that the intermolecular bands are almost flat and half-filled and that the TCNQ/graphene/Ru(0001) system might be a realization of the flat-band Hubbard ferromagnets.

Our theoretical study provides a further route in search of flat-band ferromagnets: For this purpose one can use not only Mielke's or Tasaki's flat-band systems $., 9,9$ Also flat-band systems which do not show ground-state FM for ideal geometry are promising candidates, since one may expect that deviations from the perfect flat-band geometry are always present in experimental realizations of such systems.

\section{Acknowledgments}

We are grateful to M. Maksymenko for discussions. The numerical calculations were performed using J. Schulenburg's spinpack ${ }^{56}$ The present study was supported by the DFG (project RI615/21-1). J. R. and O. D. would like to acknowledge the hospitality of the MPIPKS, Dresden in October-December of 2013. O. D. would like to thank the Abdus Salam International Centre for Theo- 
1 D. N. Sheng, Z.-C. Gu, K. Sun, and L. Sheng, Nat. Commun. 2, 389 (2011); E. Tang, J.-W. Mei, and X.G. Wen, Phys. Rev. Lett. 106, 236802 (2011); K. Sun, Z. Gu, H. Katsura, and S. Das Sarma, Phys. Rev. Lett. 106, 236803 (2011); T. Neupert, L. Santos, C. Chamon, and C. Mudry, Phys. Rev. Lett. 106, 236804 (2011); E. Bergholtz and Zhao Liu, Int. J. Mod. Phys. B 27, 1330017 (2013).

2 H. Katsura, I. Maruyama, A. Tanaka, and H. Tasaki, Europhys. Lett. 91, 57007 (2010).

3 C. Wu, D. Bergman, L. Balents, and S. Das Sarma, Phys. Rev. Lett. 99, 070401 (2007); Y.-F. Wang, Z.-C. Gu, C.D. Gong, and D. N. Sheng, Phys. Rev. Lett. 107, 146803 (2011).

4 Z. Gulácsi, A. Kampf, and D. Vollhardt, Phys. Rev. Lett. 105, 266403 (2010).

5 J. Schulenburg, A. Honecker, J. Schnack, J. Richter, and H.-J. Schmidt, Phys. Rev. Lett. 88, 167207 (2002); J. Richter, J. Schulenburg, A. Honecker, J. Schnack, and H.-J. Schmidt, J. Phys.: Condens. Matter 16, S779 (2004); J. Richter, O. Derzhko, and J. Schulenburg, Phys. Rev. Lett. 93, 107206 (2004).

6 D. Leykam, S. Flach, O. Bahat-Treidel, and A. S. Desyatnikov, Phys. Rev. B 88, 224203 (2013); S. Flach, D. Leykam, J. D. Bodyfelt, P. Matthies, and A. S. Desyatnikov, Europhys. Lett. 105, 30001 (2014).

7 D. C. Tsui, H. L. Störmer, and A. C. Gossard, Phys. Rev. Lett. 48, 1559 (1982); H. L. Störmer, Rev. Mod. Phys. 71, 875 (1999).

8 A. Mielke, J. Phys. A 24, L73 (1991); 24, 3311 (1991); 25, 4335 (1992); Phys. Lett. A 174, 443 (1993).

${ }^{9}$ H. Tasaki, Phys. Rev. Lett. 69, 1608 (1992).

10 A. Mielke and H. Tasaki, Commun. Math. Phys. 158, 341 (1993).

11 H. Tasaki, Prog. Theor. Phys. 99, 489 (1998).

12 T. Okubo, M. Yamada, A. Thamizhavel, S. Kirita, Y. Inada, R. Settai, H. Harima, K. Takegahara, A. Galatanu, E. Yamamoto, and Y. Onuki, J. Phys.: Condens. Matter 15, L721 (2003); Z. Gulácsi, A. Kampf, and D. Vollhardt, Phys. Rev. Lett. 99, 026404 (2007).

13 M. Maksymenko, A. Honecker, R. Moessner, J. Richter, and O. Derzhko, Phys. Rev. Lett. 109, 096404 (2012).

14 H. Tasaki, Phys. Rev. Lett. 73, 1158 (1994); 75, 4678 (1995); Commun. Math. Phys. 242, 445 (2003).

15 K. Kusakabe and H. Aoki, Phys. Rev. Lett. 72, 144 (1994); H. Aoki, Int. J. Mod. Phys. B 17, 4953 (2003).

16 K. Kusakabe and H. Aoki, Physica B 194-196, 215 (1994).

17 H. Tasaki, J. Stat. Phys. 84, 535 (1996).

18 Y. Watanabe and S. Miyashita, J. Phys. Soc. Jpn. 66, 2123 (1997); 66, 3981 (1997); 68, 3086 (1999); R. Arita and H. Aoki, Phys. Rev. B 61, 12261 (2000).

19 A. Tanaka and T. Idogaki, J. Phys. Soc. Jpn. 67, 401 (1998).

20 A. Mielke, Phys. Rev. Lett. 82, 4312 (1999); J. Phys. A 32, 8411 (1999).

21 A. Tanaka and T. Idogaki, Physica A 297, 441 (2001).

22 A. Tanaka and H. Ueda, Phys. Rev. Lett. 90, 067204 (2003).
23 T. Sekizawa, J. Phys. A 36, 10451 (2003).

24 C. D. Batista and B. S. Shastry, Phys. Rev. Lett. 91, 116401 (2003).

${ }^{25}$ H. Ueda, A. Tanaka, and T. Idogaki, J. Magn. Magn. Mater. 272-276, 950 (2004).

${ }^{26}$ H. Ueda and T. Idogaki, Phys. Rev. B 69, 104424 (2004).

27 A. Tanaka and H. Tasaki, Phys. Rev. Lett. 98, 116402 (2007).

28 Z. Gulácsi, A. Kampf, and D. Vollhardt, Prog. Theor. Phys. Suppl. 176, 1 (2008); R. Trencsényi, E. Kovács, and Z. Gulácsi, Philos. Mag. 89, 1953 (2009); R. Trencsényi and Z. Gulácsi, Eur. Phys. J. B 75, 511 (2010); Z. Gulácsi, Int. J. Mod. Phys. B 27, 1330009 (2013).

29 O. Derzhko, A. Honecker, and J. Richter, Phys. Rev. B 76, 220402 (2007); O. Derzhko, J. Richter, A. Honecker, M. Maksymenko, and R. Moessner, Phys. Rev. B 81, 014421 (2010).

30 H. Tasaki, Eur. Phys. J. B 64, 365 (2008).

31 O. Derzhko, A. Honecker, and J. Richter, Phys. Rev. B 79, 054403 (2009).

32 L. Lu, J. Phys. A 42, 265002 (2009).

33 M. Maksymenko, O. Derzhko, and J. Richter, Eur. Phys. J. B 84, 397 (2011).

34 A. Mielke, Eur. Phys. J. B 85, 184 (2012).

35 H. Tamura, K. Shiraishi, T. Kimura, and H. Takayanagi, Phys. Rev. B 65, 085324 (2002); T. Kimura, H. Tamura, K. Shiraishi, and H. Takayanagi, Phys. Rev. B 65, 081307 (2002); M. Ichimura, K. Kusakabe, S. Watanabe, and T. Onogi, Phys. Rev. B 58, 9595 (1998); H. Ishii, T. Nakayama, and J.-i. Inoue, Phys. Rev. B 69, 085325 (2004).

36 S. Nishino, M. Goda, and K. Kusakabe, J. Phys. Soc. Jpn. 72, 2015 (2003); S. Nishino and M. Goda, J. Phys. Soc. Jpn. 74, 393 (2005).

37 R. Arita, Y. Suwa, K. Kuroki, and H. Aoki, Phys. Rev. Lett. 88, 127202 (2002); Y. Suwa, R. Arita, K. Kuroki, and H. Aoki, Phys. Rev. B 68, 174419 (2003); H. Aoki, Applied Surface Science 237, 2 (2004).

38 H.-H. Lin, T. Hikihara, H.-T. Jeng, B.-L. Huang, C.Y. Mou, and X. Hu, Phys. Rev. B 79, 035405 (2009).

39 M. Garnica, D. Stradi, S. Barja, F. Calleja, C. Diaz, M. Alcami, N. Martin, A. L. V. de Parga, F. Martin, and R. Miranda, Nat. Phys. 9, 368 (2013).

40 I. Bloch, Nat. Phys. 1, 23 (2005).

41 I. Bloch, J. Dalibard, and W. Zwerger, Rev. Mod. Phys. 80, 885 (2008).

42 G.-W. Chern, C.-C. Chien, and M. Di Ventra, arXiv:1307.6128.

43 M. E. Zhitomirsky and H. Tsunetsugu, Phys. Rev. B 70, 100403(R) (2004); 75, 224416 (2007).

44 O. Derzhko and J. Richter, Phys. Rev. B 70, 104415 (2004); Eur. Phys. J. B 52, 23 (2006).

45 M. Maksymenko, R. Moessner, and K. Shtengel, arXiv:1401.6172.

46 J. Villain, R. Bidaux, J. P. Carton, and R. Conte, J. Phys. 41, 1263 (1980).

47 E. F. Shender, Zh. Eksp. Teor. Fiz. 83, 326 (1982) [Sov. Phys. JETP 56, 178 (1982)]. 
48 H.-J. Schmidt, J. Richter, and R. Moessner, J. Phys. A. 39, 10673 (2006).

${ }^{49}$ H. Kikuchi, Y. Fujii, M. Chiba, S. Mitsudo, T. Idehara, T. Tonegawa, K. Okamoto, T. Sakai, T. Kuwai, and H. Ohta, Phys. Rev. Lett. 94, 227201 (2005); H. Jeschke, I. Opahle, H. Kandpal, R. Valenti, H. Das, T. SahaDasgupta, O. Janson, H. Rosner, A. Brühl, B. Wolf, M. Lang, J. Richter, S. Hu, X. Wang, R. Peters, T. Pruschke, and A. Honecker, Phys. Rev. Lett. 106, 217201 (2011).

50 D. J. Klein, J. Chem. Phys. 61, 786 (1974).

51 P. Fulde, Electron Correlations in Molecules and Solids (Springer-Verlag, Berlin, Heidelberg, 1993), p. 77.

52 F. H. L. Essler, H. Frahm, F. Göhmann, A. Klümper, and V. E. Korepin, The One-Dimensional Hubbard Model
(Cambridge University Press, Cambridge, UK, 2005), p. 38 .

53 A. B. Harris and R. V. Lange, Phys. Rev. 157, 295 (1967).

54 X. Mo, K. M. S. Etheredge, S.-J. Hwu, and Q. Huang, Inorg. Chem. 45, 3478 (2006); R. A. Mole, J. A. Stride, P. F. Henry, M. Hoelzel, A. Senyshyn, A. Alberola, C. J. G. Garcia, P. R. Raithby, and P. T. Wood, Inorg. Chem. 50, 2246 (2011).

55 M. Ishii, H. Tanaka, M. Hori, H. Uekusa, Y. Ohashi, K. Tatani, Y. Narumi, and K. Kindo, J. Phys. Soc. Jpn. 69, 340 (2000); A. Honecker and A. Läuchli, Phys. Rev. B 63, 174407 (2001).

56 http://www-e.uni-magdeburg.de/jschulen/spin/

\section{Appendix: Perturbation theory for the 5-site two-electron problem}

In this Appendix we present some details of the perturbation-theory calculations for the 5 -site two-electron problem which are used in the main text.

We split the Hamiltonian of the model $H$ given in Eq. (1) for the 5-site cluster into the main part

$$
\begin{aligned}
& \mathrm{H}_{0}=\sum_{\sigma=\uparrow, \downarrow}\left[t_{2}\left(c_{m, 1, \sigma}^{\dagger} c_{m, 2, \sigma}+c_{m, 2, \sigma}^{\dagger} c_{m, 1, \sigma}\right)+t_{2}\left(c_{m+1,1, \sigma}^{\dagger} c_{m+1,2, \sigma}+c_{m+1,2, \sigma}^{\dagger} c_{m+1,1, \sigma}\right)\right] \\
& +U\left(n_{m, 1, \uparrow} n_{m, 1, \downarrow}+n_{m, 2, \uparrow} n_{m, 2, \downarrow}+n_{m, 3, \uparrow} n_{m, 3, \downarrow}+n_{m+1,1, \uparrow} n_{m+1,1, \downarrow}+n_{m+1,2, \uparrow} n_{m+1,2, \downarrow}\right)
\end{aligned}
$$

and the perturbation

$$
\begin{gathered}
\mathrm{V}=\sum_{\sigma=\uparrow, \downarrow}\left[t_{3}\left(c_{m, 1, \sigma}^{\dagger} c_{m, 3, \sigma}+c_{m, 3, \sigma}^{\dagger} c_{m, 1, \sigma}\right)+t_{1}\left(c_{m, 2, \sigma}^{\dagger} c_{m, 3, \sigma}+c_{m, 3, \sigma}^{\dagger} c_{m, 2, \sigma}\right)\right. \\
\left.+t_{1}\left(c_{m, 3, \sigma}^{\dagger} c_{m+1,1, \sigma}+c_{m+1,1, \sigma}^{\dagger} c_{m, 3, \sigma}\right)+t_{3}\left(c_{m, 3, \sigma}^{\dagger} c_{m+1,2, \sigma}+c_{m+1,2, \sigma}^{\dagger} c_{m, 3, \sigma}\right)\right] .
\end{gathered}
$$

Using as a complete set of the one-electron states the states $l_{m, \sigma}^{\dagger}|0\rangle, l_{m+1, \sigma}^{\dagger}|0\rangle, c_{m, 3, \sigma}^{\dagger}|0\rangle, d_{m, \sigma}^{\dagger}|0\rangle, d_{m+1, \sigma}^{\dagger}|0\rangle(\sigma=\uparrow, \downarrow)$ with $l_{m, \sigma}^{\dagger}=\left(c_{m, 1, \sigma}^{\dagger}-c_{m, 2, \sigma}^{\dagger}\right) / \sqrt{2}, d_{m, \sigma}^{\dagger}=\left(c_{m, 1, \sigma}^{\dagger}+c_{m, 2, \sigma}^{\dagger}\right) / \sqrt{2}$, we find all 45 eigenstates $|\alpha\rangle$ and their energies $\mathrm{E}_{\alpha}$ of the unperturbed Hamiltonian $\mathrm{H}_{0}$ (A1) in the two-electron subspace. The ground state of the unperturbed Hamiltonian $\mathrm{H}_{0}$ in the two-electron subspace $|\mathrm{GS}\rangle$ is four-fold degenerate, i.e., consists of the 3 triplet states, $|t, 1\rangle=l_{m, \uparrow}^{\dagger} l_{m+1, \uparrow}^{\dagger}|0\rangle$, $|t, 0\rangle=(1 / \sqrt{2})\left(l_{m, \uparrow}^{\dagger} l_{m+1, \downarrow}^{\dagger}+l_{m, \downarrow}^{\dagger} l_{m+1, \uparrow}^{\dagger}\right)|0\rangle,|t,-1\rangle=l_{m, \downarrow}^{\dagger} l_{m+1, \downarrow}^{\dagger}|0\rangle$, and the singlet state, $|s\rangle=(1 / \sqrt{2})\left(l_{m, \uparrow}^{\dagger} \dagger_{m+1, \downarrow}^{\dagger}-\right.$ $\left.l_{m, \downarrow}^{\dagger} l_{m+1, \uparrow}^{\dagger}\right)|0\rangle$, with the ground-state energy $\mathrm{E}_{\mathrm{GS}}=E_{t}^{(0)}=E_{s}^{(0)}=-2 t_{2}$.

The lowest-order perturbation-theory corrections to the ground-state energy $\mathrm{E}_{\mathrm{GS}}$ are as follows:

$$
\begin{array}{r}
E_{\mathrm{GS}}^{(2)}=\sum_{\alpha}^{\prime} \frac{\langle\mathrm{GS}|\mathrm{V}| \alpha\rangle\langle\alpha|\mathrm{V}| \mathrm{GS}\rangle}{\mathrm{E}_{\mathrm{GS}}-\mathrm{E}_{\alpha}}, \\
E_{\mathrm{GS}}^{(3)}=\sum_{\alpha}^{\prime} \sum_{\beta}^{\prime} \frac{\langle\mathrm{GS}|\mathrm{V}| \alpha\rangle\langle\alpha|\mathrm{V}| \beta\rangle\langle\beta|\mathrm{V}| \mathrm{GS}\rangle}{\left(\mathrm{E}_{\mathrm{GS}}-\mathrm{E}_{\alpha}\right)\left(\mathrm{E}_{\mathrm{GS}}-\mathrm{E}_{\beta}\right)}, \\
E_{\mathrm{GS}}^{(4)}=\sum_{\alpha}^{\prime} \sum_{\beta}^{\prime} \sum_{\gamma}^{\prime} \frac{\langle\mathrm{GS}|\mathrm{V}| \alpha\rangle\langle\alpha|\mathrm{V}| \beta\rangle\langle\beta|\mathrm{V}| \gamma\rangle\langle\gamma|\mathrm{V}| \mathrm{GS}\rangle}{\left(\mathrm{E}_{\mathrm{GS}}-\mathrm{E}_{\alpha}\right)\left(\mathrm{E}_{\mathrm{GS}}-\mathrm{E}_{\beta}\right)\left(\mathrm{E}_{\mathrm{GS}}-\mathrm{E}_{\gamma}\right)}-\sum_{\alpha}^{\prime} \sum_{\beta}^{\prime} \frac{\langle\mathrm{GS}|\mathrm{V}| \alpha\rangle\langle\alpha|\mathrm{V}| \mathrm{GS}\rangle\langle\mathrm{GS}|\mathrm{V}| \beta\rangle\langle\beta|\mathrm{V}| \mathrm{GS}\rangle}{\left(\mathrm{E}_{\mathrm{GS}}-\mathrm{E}_{\alpha}\right)^{2}\left(\mathrm{E}_{\mathrm{GS}}-\mathrm{E}_{\beta}\right)} .
\end{array}
$$

Here the superscript prime means that the sum extends over all states of the unperturbed Hamiltonian $\mathrm{H}_{0}$ except the ground states. After straightforward calculations on the base of Eqs. (A3) and (A2) we find the following nonzero corrections to the ground-state energy:

$$
E_{t}^{(2)}=E_{s}^{(2)}=-\frac{\left(t_{3}-t_{1}\right)^{2}}{t_{2}}
$$

and

$$
E_{t}^{(4)}=-\frac{\left(t_{3}+t_{1}\right)^{2}\left(t_{3}-t_{1}\right)^{2}}{2 t_{2}^{3}}+\frac{\left(t_{3}-t_{1}\right)^{4}}{t_{2}^{3}}
$$




$$
\begin{array}{r}
E_{s}^{(4)}(U)=-\frac{\left(t_{3}+t_{1}\right)^{2}\left(t_{3}-t_{1}\right)^{2}}{4 t_{2}^{3}}+\frac{\left(t_{3}-t_{1}\right)^{4}}{t_{2}^{3}} \\
-\frac{a^{2}\left(t_{3}-t_{1}\right)^{4}}{\left(2 t_{2}+\frac{U}{2}-\sqrt{\frac{U^{2}}{4}+4 t_{2}^{2}}\right) t_{2}^{2}}-\frac{\left(t_{3}+t_{1}\right)^{2}\left(t_{3}-t_{1}\right)^{2}}{2\left(2 t_{2}+U\right) t_{2}^{2}}-\frac{2\left(t_{3}-t_{1}\right)^{4}}{\left(2 t_{2}+U\right) t_{2}^{2}}-\frac{b^{2}\left(t_{3}-t_{1}\right)^{4}}{\left(2 t_{2}+\frac{U}{2}+\sqrt{\frac{U^{2}}{4}+4 t_{2}^{2}}\right) t_{2}^{2}}, \\
a^{2}=\frac{1}{C_{-}^{2}}\left(\frac{U}{4 t_{2}}-\sqrt{\frac{U^{2}}{16 t_{2}^{2}}+1}-1\right)^{2}, \quad b^{2}=\frac{1}{C_{+}^{2}}\left(\frac{U}{4 t_{2}}+\sqrt{\frac{U^{2}}{16 t_{2}^{2}}+1}-1\right)^{2}, \\
C_{\mp}^{2}=\left(\frac{U}{4 t_{2}} \mp \sqrt{\frac{U^{2}}{16 t_{2}^{2}}+1}-1\right)^{2}+\left(\frac{U}{4 t_{2}} \mp \sqrt{\frac{U^{2}}{16 t_{2}^{2}}+1}+1\right)^{2},
\end{array}
$$

i.e., the ground-state degeneracy is lifted in the fourth order of the perturbation theory.

The triplet-state energy does not depend on $U$; the obtained results (A4), (A5) are given in Eq. (3). In the limit $U \rightarrow \infty$, we have $U / 2-\sqrt{U^{2} / 4+4 t_{2}^{2}} \rightarrow 0, a^{2} \rightarrow 1 / 2$ and the singlet-state energy $E_{s}(\infty)$ is given by the formula in Eq. (4). In the small- $U$ limit, we have $\sqrt{U^{2} / 4+4 t_{2}^{2}} \rightarrow 2 t_{2}, a^{2} \rightarrow 1, b^{2} \rightarrow 0$ and the dominating term in $E_{s}(U)$ (A6) is $-2\left(t_{3}-t_{1}\right)^{4} /\left(U t_{2}^{2}\right)$.

Using Eqs. (A5) and (A6), the equation for $U_{c}, E_{t}=E_{s}\left(U_{c}\right)$, can be written as follows:

$$
\left(\frac{t_{3}-t_{1}}{t_{3}+t_{1}}\right)^{2}=\frac{\frac{U_{c}^{2}}{t_{2}^{2}}}{\frac{U_{c}^{2}}{t_{2}^{2}}+18 \frac{U_{c}}{t_{2}}+16}
$$

Solving Eq. (A7) with respect to $U_{c} / t_{2}$ we get Eq. (5). 